\title{
Application Research of Digital Signal Processing Course Based on Matlab Teaching
}

\author{
Wang Yuanmin ${ }^{1,3}$, Zhang $\mathrm{Li}^{2,3}$ \\ 1.Leshan Vocational and Technical College, Leshan Sichuan, 614000,China \\ 2.Leshan Normal University,Leshan Sichuan, 614000, China \\ 3.Chengdu University of Technology,Leshan Sichuan, 610059, China
}

\begin{abstract}
In the course of digital signal processing, MATLAB software can be used in combination to change the traditional teaching form, to show students rich teaching content, to stimulate students' interest in learning and to improve teaching quality.This paper first introduces the characteristics of digital signal processing course, and then analyzes the advantages of MATLAB software and digital signal processing course. The application of MATLAB software in digital signal processing course teaching is explored in detail in order to provide reference for teaching practice.
\end{abstract}

Keywords: digital signal processing; characteristics; Matlab; teaching

Digital signal processing technology can be applied in many technical fields, including communication engineering, industrial production, national defense military industry and so on, which plays a very important role in promoting social and economic development.In the teaching of digital signal processing course, the algorithm should be regarded as the core, the theory is very strong, a lot of professional knowledge is abstract, in the theoretical derivation, it is necessary to master the rich mathematical foundation. Therefore, in the course of digital signal processing, it is difficult for students to understand. In the process of teaching, we should not only impart rich basic theoretical knowledge

https://doi.org/10.47852/bonviewCETR2020010221

This is an open access article published by the BON VIEW PUBLISHING PTE. under the Creative Commons Attributions License. 
to students, but also strengthen the cultivation of skills. Guide students to improve their interest in learning and creative consciousness. On this basis, teachers need to innovate and adjust the teaching mode of digital signal processing course, while MATLAB is high performance software with visual characteristics. By applying the MATLAB to the teaching of digital information processing course, the abstract theoretical knowledge is taught to the students by visualization, and the demonstration and analysis are carried out.At the same time, the combination of experimental teaching and afterclass exercises can help students better understand and master the professional knowledge of digital information processing courses and the methods of digital information processing.

\section{Characteristics of digital signal processing courses}

In the course of digital information processing, many concepts are abstract, students' understanding is difficult, and the calculation is large. The mathematical calculation is required as the basis, the derivation formula is complex, the logic is strong, and the correlation degree of the course before and after is relatively high.However, in the actual teaching, the level of mathematics knowledge of many students is uneven, and the understanding of the previous courses generally stays at the surface level, which can not fully master the theoretical knowledge of digital information processing. In addition, in the course teaching of digital information processing, the course hours are limited, and some teachers have improper control of teaching progress, so it is difficult to cultivate students' logical thinking ability. As a result, students gradually lose interest in digital information processing courses. The teaching task of digital information processing course is heavy and the teaching time is small. Teachers should apply MATLAB software to the teaching of MATLAB software course to ensure the high efficiency of digital information processing course teaching ${ }^{[1]}$.

\section{Integration of Matlab applications and digital signal processing courses}

MATLAB software has great influence and is a computer language developed from matrix operation. It has the advantage of high integration. In the practical application of MATLAB software, the operation mode is flexible and convenient.It can also provide graphic visualization function, interface design function, language interface function and so on. It has been widely used in many fields, such as numerical calculation, engineering drawing, control system design, digital signal processing and so on. MATLAB software has high programming efficiency and can provide toolbox for special fields. Many common algorithms in signal processing toolbox have corresponding functions, such as FFT function, which can be used to design FIR digital filter.

In the course of digital signal processing, the knowledge points are abundant, and many concepts 
are abstract, so it is difficult for students to understand.In the teaching activities, the MATLAB software can be integrated, the classical analysis and processing methods can be displayed by computer, and the theoretical knowledge and practical operation can be effectively combined, so that the students can gradually master the signal analysis and processing methods.After completing the teaching of each chapter, the teacher should arrange the software programming homework for the students according to the teaching content, make full use of the signal processing function of the MATLAB software, apply the computer connection, and gradually master the rich knowledge of digital signal processing.

\section{Teaching Ideas of Digital Signal Processing Course Based on Matlab}

A. Set up the teaching content reasonably.

In the course of digital signal processing, there are many formulas, which involve a wide range of knowledge and short hours. In the actual teaching.It is necessary to analyze the teaching requirements and professional characteristics, to determine the key points and difficulties in the course teaching, to impart basic concepts and theoretical knowledge to the students, to emphasize the curriculum ideas, and to weaken the derivation of the formula appropriately.For example, in the teaching of fast Fourier transform, this knowledge point is very important, but the calculation formula and principle are more complex, MATLAB the software has the fft command, through the teaching of fast Fourier transform is carried out, it is convenient to carry out teaching activities.In the concrete teaching process, it is necessary to introduce the concept and characteristics of fast Fourier transform the students first, and then guide the students to solve all kinds of problems by applying the fft commands in the MATLAB software, so that the students can absorb the knowledge points better.

B. Adopting visual classroom teaching methods.

In the digital signal analysis and processing, many formulas need to be applied, but the students' understanding of all kinds of formulas is more difficult, which requires innovative teaching methods, so that students can intuitively understand the professional concepts, calculation formulas and so on.Using multimedia technology, we can display theoretical knowledge and graphics for students by means of graphics and video, and improve the diversity, interest and visual characteristics of classroom teaching. MATLAB software can call the function library, carry on the simulation analysis to the basic theory knowledge, display the course teaching content in the form of image, animation and so on, and the detailed rock digital signal analysis and processing process, and respond to the result, so as to facilitate the students to understand, and expand the students' thinking space at the same time.

C. Pay attention to practical teaching.

In the course of digital signal processing, students are required to master solid basic knowledge 
of mathematics. For a large number of theories and conclusions, digital derivation is needed. In this process, it is difficult for students to understand the essence of each formula and theory.In this regard, in the course of teaching, students should emphasize the physical meaning and application methods, and adopt scientific research practice teaching activities to help students deepen their understanding and application of image processing and language signal processing. To innovate the teaching method of digital signal processing course, DSP devices or application MATLAB software can be used. Among them, MATLAB has the function of algorithm simulation, which can demonstrate and analyze the teaching content, verify convolution operation, signal filtering, Fourier analysis and so on, and make abstract content concrete image and improve learning efficiency.

D. Construct network teaching environment.

Network technology is developing rapidly. In teaching activities, network technology and abundant network resources can also be used to create course websites, and to create test questions database and electronic courseware for digital signal processing course teaching. In the process of learning, students can use the network platform to download courseware in time, and use extracurricular time to learn. At the same time, they can also create a virtual laboratory for digital signal processing and analysis, which is convenient for teachers and students to communicate and share their experience in the learning process in time.

E. Classroom homework and examination reform.

The concept of digital signal processing course is rich, and abstract is difficult to understand. It is necessary to apply a lot of consensus in numerical calculation, and the calculation process is complex. At the same time, teachers will be limited in arranging homework for students, which can not guarantee the teaching quality.Therefore, in the innovation of teaching mode, teachers can use MATLAB software to assist teaching, and arrange digital signal processing homework set for students, MATLAB the signal processing function of software is powerful.During the learning process, students can use computer and MATLAB software to practice exercises, better understand the digital signal processing and analysis, according to which homework exercises, efficient completion of teaching tasks.In the course of teaching reform and innovation, we should reasonably determine the teaching objectives of digital signal processing courses of digital signal processing course according to the concrete content of teaching reform plan.In view of the students' learning process, including curriculum design, experimental practice teaching and so on, it is necessary to carry on the examination, to change the deficiency of emphasizing theoretical teaching and neglecting practical teaching in nursing teaching. In the evaluation of students' academic performance, it is necessary not only to check the students' final examination results, but also to examine and analyze the learning situation in the course of teaching, 
including classroom notes, classroom tests, experimental results, etc ${ }^{[2]}$ 。

\section{Application of MATLAB in the Teaching of Digital Signal Processing}

A. Frequency domain analysis of discrete signal systems.

In general, the time domain shape is generally used to display the signal. In the time domain analysis, the time axis should be taken as the coordinate parameter, and the signal should be analyzed accordingly. The signal has frequency domain characteristics, and the characteristics of the signal can be analyzed by taking the frequency axis as the parameter. When the signal is changed from time domain analysis to frequency domain analysis, Fourier transform should be applied.In the practical application of Fourier transform, for time domain signal $x(t)$, can be transformed into frequency domain signal $x$ (f). After Fourier transform is realized, the signal characteristics can be analyzed from other angles. Then help analysts to fully analyze and understand the signal.During the frequency domain analysis of discrete systems, MATLAB software commands can be used to gradually stack sine waves from low frequency to high frequency, gradually form square waves, draw waveforms and synthesize them. Students can observe and understand the theory of superimposed sine wave approximation square wave intuitively and deepen their understanding of signal time domain analysis and frequency domain analysis.In addition, it is necessary to explain the Fourier transform algorithm and the fast Fourier transform algorithm in detail, and emphasize the MATLAB software commands in two forms of transformation. At the same time, we can choose cases to analyze them to help students remember better. The frequency response curve of discrete system signal is drawn by freqz command in MATLAB software.At the same time, the zero pole distribution of the system function is drawn by using the zplane command, and the graphic analysis method is combined to help the students better understand the discrete signal analysis method ${ }^{[3]}$.

B. Filter design

In the course of digital signal processing, filter design is a very important teaching content. For digital filter, it can be divided into many types according to the characteristics of passband.The filter includes low-pass filter, band-pass filter, high-pass filter and band-stop filter. In addition, according to the unit impulse response, it can be divided into two types: IIR digital filter and FIR digital filter.Among them, in the practical application of IIR digital filter, it is necessary to create and design according to the knowledge of professional analog filter and design chart.The finite length unit impulse response $h(n)$ can be applied in the practical application of FIR digital filter to ensure that it can meet the requirements of technical index control.The related concepts of filter are more complex, students understand more difficult, in the specific design process, the design principles involved are also more complex, so it 
is difficult for students to understand.Therefore, in this part of the teaching process, students should focus on MATLAB software commands and FDATool. FDATool is a very important tool type in filter design and analysis, which is powerful and includes all kinds of filter design methods in signal processing toolbox. It can effectively improve the convenience of programming. The design index should be determined in the design process of FIR band-stop filter, and then the remezord function and remez function should be applied to design the FIR band-stop filter, and the command window running FDATool, in the software should be applie.Set the index parameters in the MATLAB software interface and draw the filter graphics.By using MATLAB software to carry out teaching, students can better understand the design method and design process of filter.

\section{Conclusion}

To sum up, this paper mainly explores the application of MATLAB software in the teaching of digital signal processing.Digital signal processing course has many teaching contents, many knowledge points are abstract and complex, students' understanding is difficult, this paper puts forward the application of MATLAB software to carry out digital signal processing teaching.By creating design practice teaching links, innovating teaching methods, expanding the depth and breadth of knowledge, arousing students' interest in learning, improving students' practical ability and ensuring teaching quality.

\section{Acknowledgement}

This work is supported in part by Fund Project of Leshan Normal University: Application of Fractional Fourier Transformochemistry Data Noise Reduction and Abnormal Feature Extraction (No. Z16010)

\section{References}

[1] Xu Xiaoxia. Research on Teaching Reform of Digital Signal Processing Based on MATLAB J]. Electronic Testing,2019,409(04):139-140.

[2] congratulations. The Application of MATLAB in the Teaching of Digital Signal Processing for Electronic Major in Transition J]. University Modernization of education ,2019,6(01):89-91.

[3] Li Qiwang, Li Simin, Xia Qinghua. The Application of Matlab in the Teaching of Digital Signal Processing [J]. Science and Technology Innovation and Application :2018254(34):180-181. 